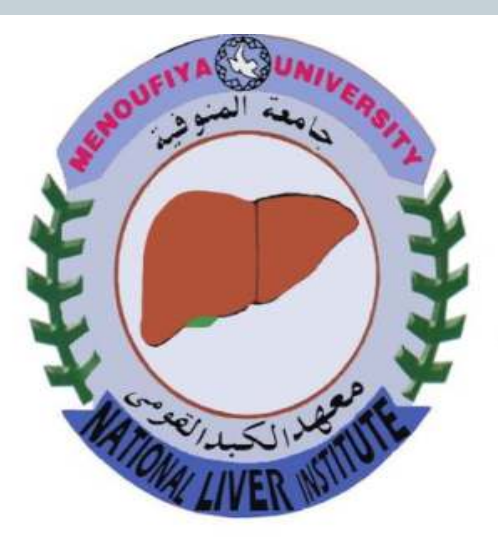

\title{
The effect of pneumoperitoneuom on systemic haemodynamic and hepatic enzymes in cirrhotic versus non cirrhotic patients undergoing laparoscopic surgery using non-invasive Electrical Cardiometry.
}

N. Hussain1, M. Othman1, M. Khalil1, S. Helal2, 1 National Liver Institute, 2 Anesthesia department . Menofia University, Egypt.

\section{Back ground \& aim of work}

Pneumoperitoneuom PP can affect hepatic perfusion and systemic hemodynamics variably.

Aim: To evaluate PP influence on haemodynamic, monitored by Electrical bio impedance Cardiometry EC (ICON, Osypka, Germany), in cirrhotic CHILD A under laparoscopic cholecystectomy LC.

\section{Materials \& Methods}

Case control study, with ethics committee approval. Consented 43 patients, ( 3 excluded turned opened). Cirrhotic C gp ( $n=20)$, non-cirrhotic NC gp $(n=20)$. Post anesthesia a central venous catheter CVP (after consent, for research only) was applied. EC readings (Heart rate HR b/min, cardiac output $\mathrm{CO}$ I/min, systemic vascular resistance SVR dyns/s/cm-5, Stroke Volume Variation SVV\%, and contractility Index $\mathrm{Cl}$ ( measures contractility, normal range: 37.5 - 62.5) Alanine aminotransferase ALT, aspartate aminotransferase AST $\mathrm{u} / \mathrm{l}$ and serum lactate $\mathrm{mg} / \mathrm{dl}$. All were reported at (T1)after induction, (T2) Post (CO2) insufflations (14-16 mmHg) and (T3) $6 \mathrm{~h}$ after surgery. Surgical complications, hospital and ICU stay were reported.

\section{Results \& Discussion}

Total insufflation and operative times/min were comparable between 2 groups. The EC measurement (CO, SVV, SVR) at all times were comparable between the 2 groups. At (T3) CVP in C gp show significant change, from base line, but still comparable with the NC gp. The median (IQR) of (Cl) in $\mathrm{C} \mathrm{gp}$ was constantly lower than NC gp through out all times of measurement and repeated measures by (Friedman test $\mathrm{P}=0.02$ ) were also significant. $\mathrm{AST}$, $\mathrm{ALT}$, blood lactate, postoperative complications, ICU and hospital stay all were also comparable between 2 groups.

\section{Conclusion}

NO major hemodynamics nor splanchnic circulation changes happen in CHILD A cirrhotic patients undergoing LC. The standard anesthesia monitors are fair enough in such procedures.The use of Cardiometry can be recommended in cirrhotic for early diagnosis of cardiomyopathy, further studies with bigger sample size is recommended.
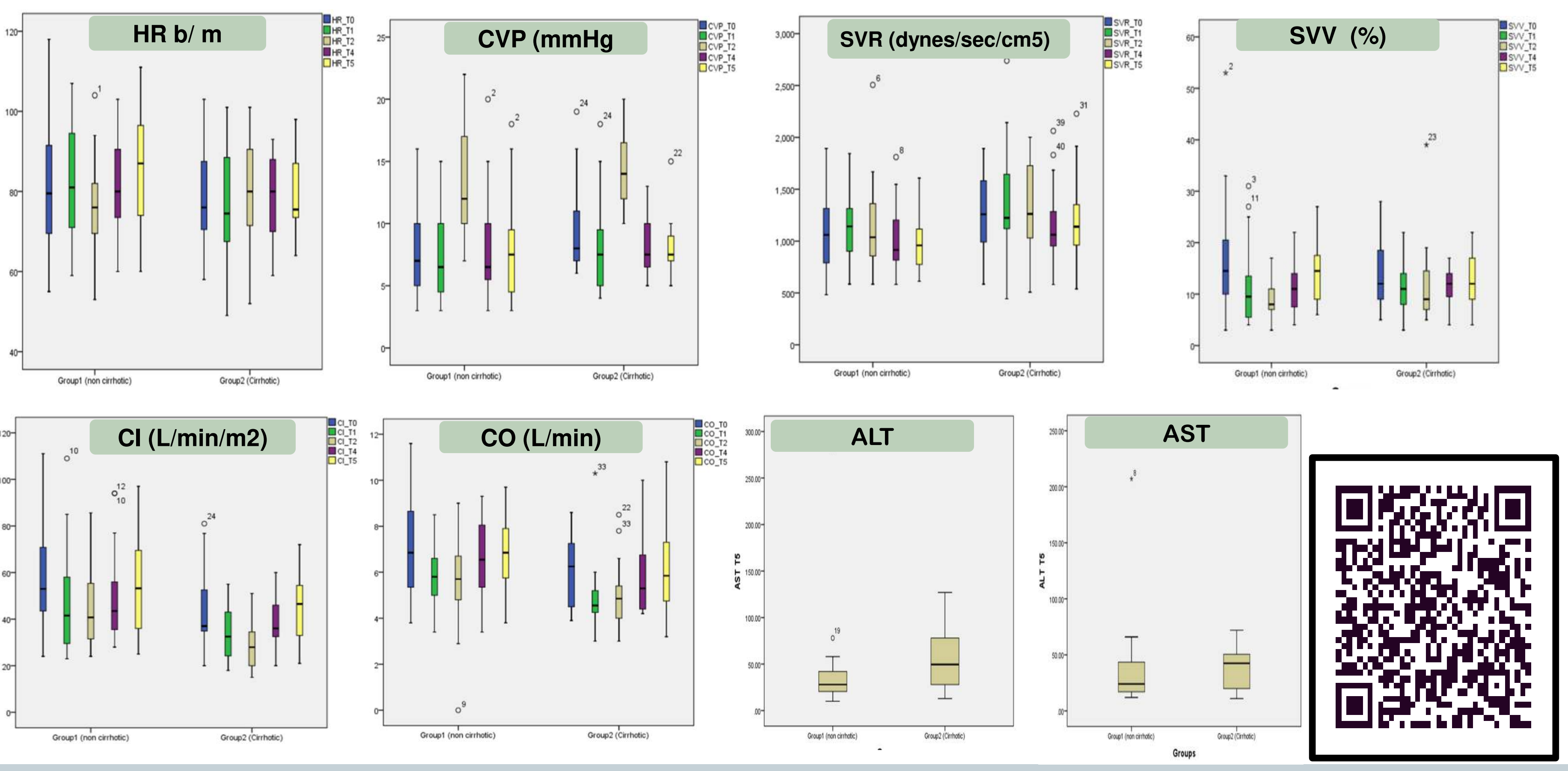